\title{
Hardware Timing Verification using KRONOS *
}

\author{
Oded Maler Sergio Yovine \\ SPECTRE - VERIMAG \\ Miniparc-ZIRST, 38330 Montbonnot, France \\ E-mail: \{Oded.Maler, Sergio.Yovine\}@imag.fr
}

\begin{abstract}
In this paper we describe the KRONOS system, a tool for verifying real-time properties based on the model of timedautomata. As an example, we show how KRONOS is applied to the verification of a MOS circuit under various delay assumptions.
\end{abstract}

\section{Introduction}

The use of finite-state automata as models of synchronous circuits is as old as computer science. Recently, verification methods for finite-state systems have proliferated from academia to industry with the progress in efficient techniques for symbolic model-checking [10], [3]. Timed automata, that is, automata augmented with fictitious clocks that can measure the time between various events, were introduced by Dill and Alur [7], [2] as models for real-time systems and asynchronous circuits.

At this real-time level of modeling, systems are not viewed anymore as generators of sequences over an abstract time domain, but rather as generators of signals over the real time domain. This allows us to speak quantitatively about the implementation (the time it takes for an instruction to be performed or for a gate to switch), the environment (the minimal time between two requests) and the requirements (the response time of the system).

The KRONOS system [4], [5], developed at VERIMAG during the last five years, represents the state-of-the-art in verification tools for real-time. It has been applied in the past

\footnotetext{
*In Proc. 7th Israeli Conference on Computer Systems and Software Engineering, June 12-13, 1996, Herzliya, Israel.
}

to the verification of a variety of time-dependent protocols.

In this paper we illustrate the application of KRONOS in another domain, namely timing analysis of hardware circuits. The theoretical basis of the translation of Boolean asynchronous circuits with delay uncertainties into timed automata has been presented in [11]. Here we apply this methodology to the verification of a MOS circuit, a problem posed to us by members of IBM Haifa research group.

The paper is organized as follows. In section 2 we give a short introduction to timed automata and real-time logics which constitute the theoretical infrastructure for KRONOS. In section 3 we describe the KRONOS system and in section 4 we demonstrate how MOS circuits are modeled as timed automata and verified using KRONOS.

\section{Theoretical Background}

\subsection{Timed Automata}

A timed automaton $\mathcal{A}$ is a tuple $\langle\mathcal{S}, \mathcal{X}, \mathcal{L}, \mathcal{E}, \mathcal{I}, \mu\rangle . \mathcal{S}$ is a finite set of locations. $\mathcal{X}$ is a finite set of real-valued variables called clocks whose values increase uniformly with time. $\mathcal{L}$ is a finite set of labels. $\mathcal{E}$ is a finite set of edges. Each edge $e$ is a tuple $\left(s, L, \psi, \rho, s^{\prime}\right)$ where $s, s^{\prime} \in \mathcal{S}, L \subseteq \mathcal{L}, \psi$ is a linear constraint on the values of the clocks expressing the timing condition requiered for the transition to be taken, and $\rho \subseteq \mathcal{X}$ is the set of clocks to be reset to 0 by the transition. $\mathcal{I}$ is a function that associates a constraint on clocks to each location. $\mu$ is a labeling of the locations with a set of atomic propositions over $\mathcal{P}$. Readers who wish at this point to see a concrete example are invited to jump to the beginning of section 4. 
A state of $\mathcal{A}$ is a pair $(s, v)$ where $v$ is a clock valuation. The state $(s, v)$ has a discrete transition to $\left(s^{\prime}, v^{\prime}\right)$, denoted $(s, v) \rightarrow^{e}\left(s^{\prime}, v^{\prime}\right)$, if $v$ satisfies the constraint $\psi$ labeling the edge $e$ and $v^{\prime}$ is such that $v^{\prime}(x)=0$ if $x \in \rho$, otherwise $v^{\prime}(x)=v(x)$. That is, discrete transitions consist in moving to another state by taking an edge whose condition is satisfied by the values of the clocks. The state $(s, v)$ has a time transition of duration $t$ to $\left(s, v^{\prime}\right)$, denoted $(s, v) \rightarrow^{t}\left(s, v^{\prime}\right)$, if $v^{\prime}(x)=v(x)+t$ for all $x \in \mathcal{X}$, and for all $t^{\prime}, 0 \leq t^{\prime} \leq t, v+t^{\prime}$ satisfies the invariant $\mathcal{I}(s)$. That is, time transitions consist in letting time progress without changing the control location. A run $r \in \mathcal{R}$ of $\mathcal{A}$ is a sequence $q_{0} \rightarrow^{t_{0}} q_{0}^{\prime} \rightarrow^{e_{0}} q_{1} \ldots$ such that $\sum_{i \geq 0} t_{i}$ diverges.

\subsection{Symbolic Verification}

KRONOS implements a symbolic verification method based on predicate transformers for computing the set of predecessors (backward analysis) and successors (forward analysis) of sets of states symbolically represented as disjunctions of convex polyhedra. A more detailed description of the symbolic verification method can be found in [9].

Backward analysis. Given a set of states $Q \subseteq \mathcal{Q}$ and an edge $e \in \mathcal{E}$, the predecessors of $Q$ by $e$ are those states that can reach a state in $Q$ by letting time elapse for some amount and then moving through $e$. We define:

$$
\operatorname{pred}_{e}(Q)=\left\{q^{\prime} \mid \exists q \in Q, t \in \mathrm{R}^{+} \cdot q^{\prime} \longrightarrow^{t} \rightarrow^{e} q\right\} .
$$

The set of all predecessors of $Q$ is $\operatorname{pred}(Q)=$ $\bigcup_{e \in \mathcal{E}} \operatorname{pred}_{e}(Q)$.

Forward analysis. Given a set of states $Q \subseteq \mathcal{Q}$ and an edge $e \in \mathcal{E}$, the successors of $Q$ by $e$ are those states that can be reached from a state in $Q$ by a time transition followed by a discrete transition through $e$. We define:

$$
\operatorname{post}_{e}(Q)=\left\{q^{\prime} \mid \exists q \in Q, t \in \mathrm{R}^{+} . q \longrightarrow^{t} \rightarrow^{e} q^{\prime}\right\} .
$$

The set of all successors of $Q$ is $p \operatorname{post}(Q)=\bigcup_{e \in \mathcal{E}} \operatorname{post}_{e}(Q)$.

\subsection{The logic TCTL}

TCTL [1, 9] is an extension of the temporal logic CTL [8] that allows reasoning about the quantitative realtime elapsed between events along the runs of a timed automaton. The formulas of TCTL are defined by the following grammar:

$$
\varphi::=p|\neg \varphi| \varphi_{1} \vee \varphi_{2}\left|\exists \diamond_{I} \varphi\right| \forall \diamond_{I} \varphi
$$

where $p \in \mathcal{P}$ is a basic predicate and $I$ is an interval. $I$ may be open or closed, bounded or unbounded. Intuitively, $\exists \diamond_{I} \varphi$ means that there exists a run and a state where $\varphi$ holds and the timed elapsed until this state since the initial state of the run belongs to the interval $I$. The formula $\forall \diamond_{I} \varphi$ means that for all runs the above property holds. We write $\forall \square \varphi$ for $\neg \exists \diamond \neg \varphi$ meaning that $\varphi$ holds in all states of all runs. The formal semantics of TCTL is given in $[1,9]$.

Model-checking consists in verifying whether a timed automaton $\mathcal{A}$ satisfies a TCTL-formula $\varphi$. Model-checking can be done symbolically using the backward and forward verification methods. We illustrate here how the methods work for only two of the temporal operators. For more details see [9].

Suppose we want to verify q qinit $\Rightarrow \exists \diamond Q$, that is, if a state in $Q$ is reachable from the initial state $q_{\text {init }}$. The backward-computation algorithm is as follows: start with $Q_{0}=Q$, and for $i \geq 0$ compute $Q_{i+1}=Q_{i} \cup \operatorname{pred}\left(Q_{i}\right)$. If $q_{\text {init }} \in Q_{i}$ for some $i$, the answer is "YES", otherwise, the answer is "NO". This procedure always terminates. A similar algorithm is used to verify $q_{\text {init }} \Rightarrow \exists \diamond_{I} Q$.

To check that the system, always remains at a set of states $Q$ we verify the formula $q_{\text {init }} \Rightarrow \forall \square Q$, or equivalently, $\neg\left(q_{\text {init }} \wedge \exists \diamond \neg Q\right)$, that is, there is no run starting at $q_{\text {init }}$ that reaches a state outside $Q$. On this formula, the forwardcomputation algorithm works as follows: start with $Q_{0}=$ $\left\{q_{\text {init }}\right\}$, and for $i \geq 0$ compute $Q_{i+1}=Q_{i} \cup \operatorname{post}\left(Q_{i}\right)$. If $Q_{i} \cap \neg Q \neq \emptyset$ for some $i$, the answer is "NO", otherwise, the answer is "YES". Notice that during the computation we can keep track of the transitions taken, so as when the answer is "NO" we can exhibit a counter-example, that is, a sequence of transitions that leads from qinit to a state in $\neg Q$.

\section{The Tool KRONOS}

KRONOS $[4,5]$ is a tool developed with the aim to assist the user to validate complex real-time systems. The tool checks whether a real-time system modeled by a timed automaton $\mathcal{A}$ satisfies a timing property specified by a TCTLformula $\varphi$. Figure 1 illustrates the structure of the tool. 


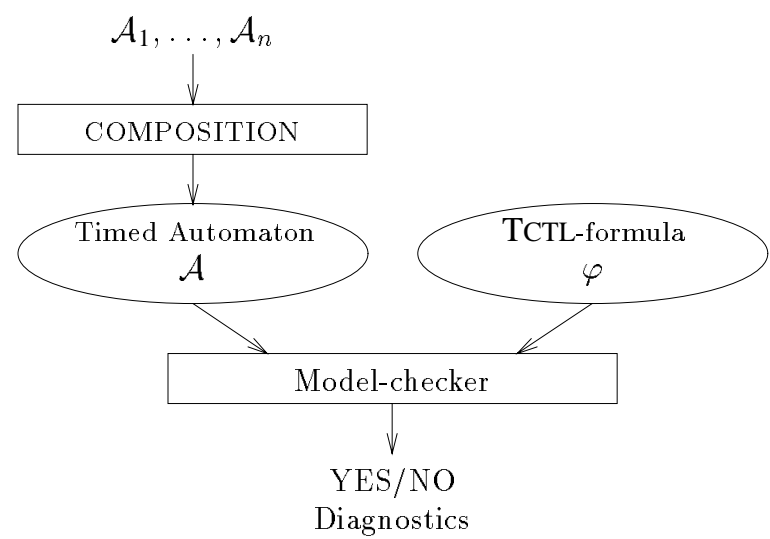

Figure 1. The KRONOS system.

A system is usually described as a set of timed automata that run in parallel and synchronise via the names labeling the edges. The parallel composition of timed automata is defined in [5]. KRONOS takes as input a set of files each one describing a single component and computes their product which is itself a timed automaton. The latter as well as the TCTL-formula to be checked are fed into the model-checker that answers "YES" whenever the formula is verified, otherwise it answers "NO" exhibiting a counterexample. The syntax of the input (automata, formulae) and output (counter-example) files, is demonstrated via examples in the appendices.

KRONOS has been used to verify a variety of protocols whose correctness strongly depends on timing parameters. Some examples of benchmarks verified with KRONOS are the Fischer's mutual exclusion protocol [6], the Tick-Tock [4] and the FDDI [6] communication protocols, and the Philips audio control protocol [5].

\section{Circuit Timing Analysis}

In this section we show how MOS circuits are modeled by a timed automata and how they are verified using KRONOS. This is not intended to be a complete nor accurate introduction to VLSI, but rather an illustration of the modeling principles.

A MOS transistor (figure 2) is a three-terminal device. It can be in two basic states $O n$ and Off. When it is On, the current flows between terminals $X_{1}$ and $X_{2}$. The transition between the two states is controlled by the switching terminal $S$. When the transistor is $O f f$ and $S$ goes up (we denote this event by $S \uparrow$ ), after a delay of 1 time unit the state of the transistor becomes $O n$. When $S$ switches back, the gate goes to Off immediately. The modeling of such a transistor as a timed automaton is rather straightforward: The transition from an Off to Rising state is triggered by the event $S \uparrow$. This transition resets the clock $C$ to zero. If an event $S \downarrow$ arrives during the interval $[0,1]$ the automaton goes back to Off. Otherwise, when $C=1$ is goes to On (emitting the signal $T \uparrow$ ) and stays there until the next $S \downarrow$ event.

Remark: We can employ other transistor models as well. For example, if a non-zero delay is associated with falling of the transitor, we would need a 4-state automaton with the additional state Falling. We can also model uncertainties in delays. The formal translation of general Boolean asynchronous circuits into timed automata is described in [11].

The circuit we consider appears in figure 3. It consists of 8 transistors and 4 input wires $P, A, B$, and $C$ which behave as follows:

- $P$ goes high immediately at start, then goes down after 20 time units, goes up again after 8 time and restarts again.

- $A$ can rise between 0 and 6 time units after start (i.e. after $P$ goes up) and falls down 16 units later.

- $B$ rises 3.3 units after $\mathrm{A}$ and falls 10 units later

- $C$ rises between 1 and 3 units after start and falls between 23.5 and 24.9 units after start.

The transistors $T_{1}, T_{5}$ and $T_{6}$ switch to $O n$ by the falling of their input signals. The value on the $X$ wire is $O n$ if current flows thru $T_{1}$ but not leaking via $T_{2}$ and $T_{3}$ or $T_{4}$. The input signals are modeled by the four timed automata in figures 4-7. State staying conditions (invariants) are written inside the states. Transitions are labeled by conditions (if not trivial), by event labels and by sets of clocks tobe reset. Note that we allow non-determinism in the behavior of the inputs. For example, when $C_{A}$ is in the interval $[0,6] A$ can either stay at $A_{0}$ or move to $A_{1}$ and generate an $A \uparrow$ transition. An example of the KRONOS source files for the input $A$ and its transistor $T_{2}$ appears in appendix 1 . 


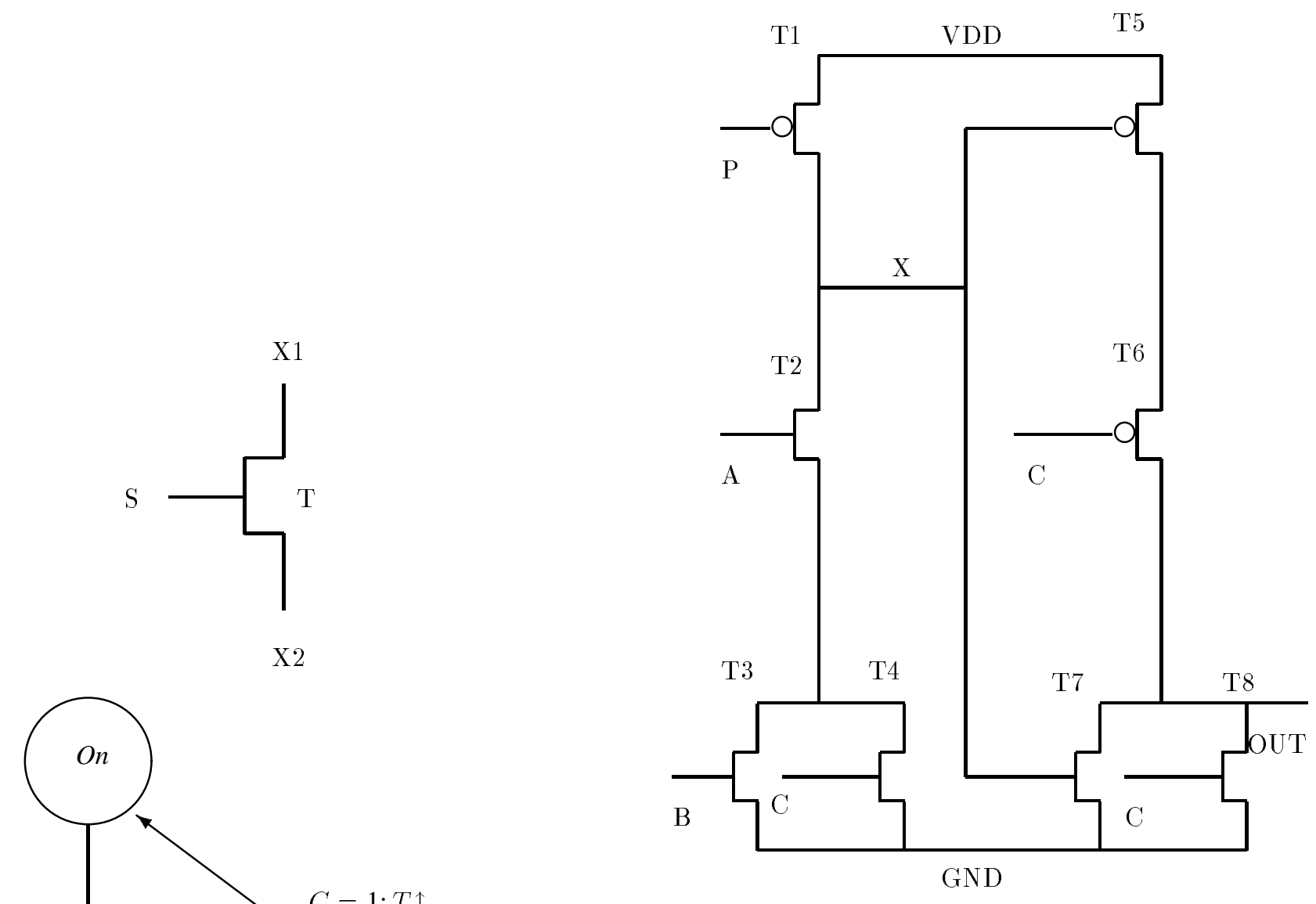

Figure 3. The circuit.

Figure 2. A transistor and its associated timed automaton.

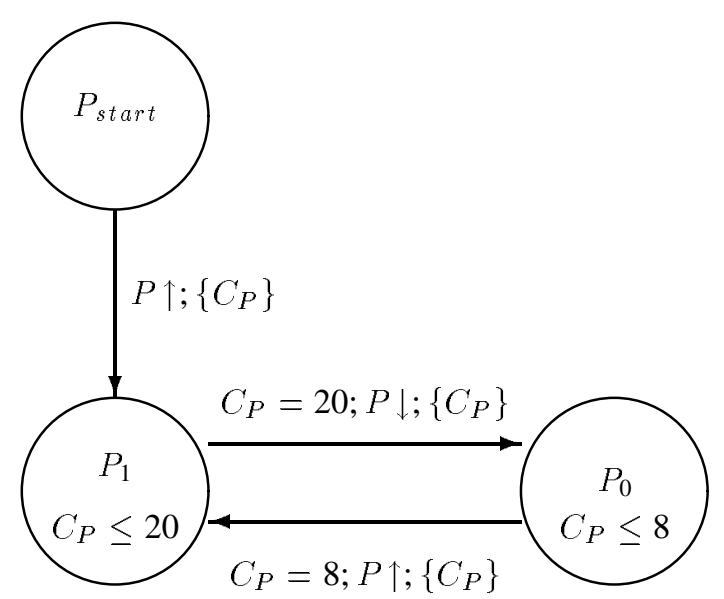

Figure 4. The automata for the input signal P. 


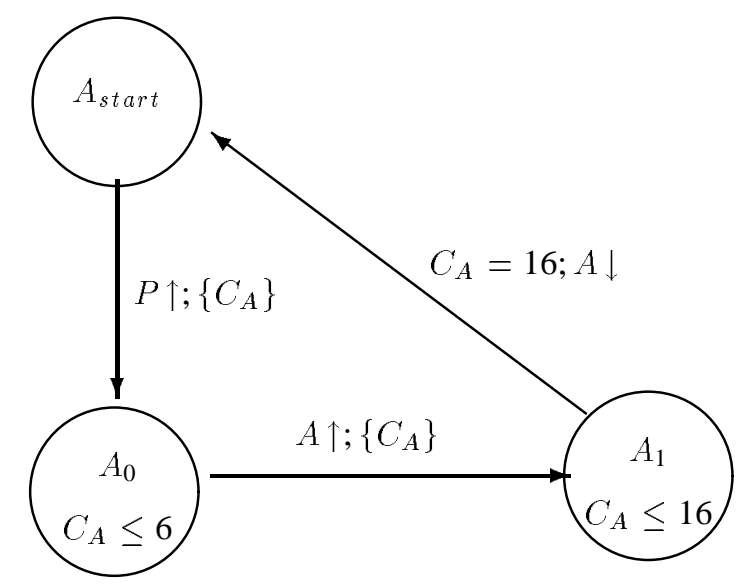

Figure 5. The automata for the input signal A.

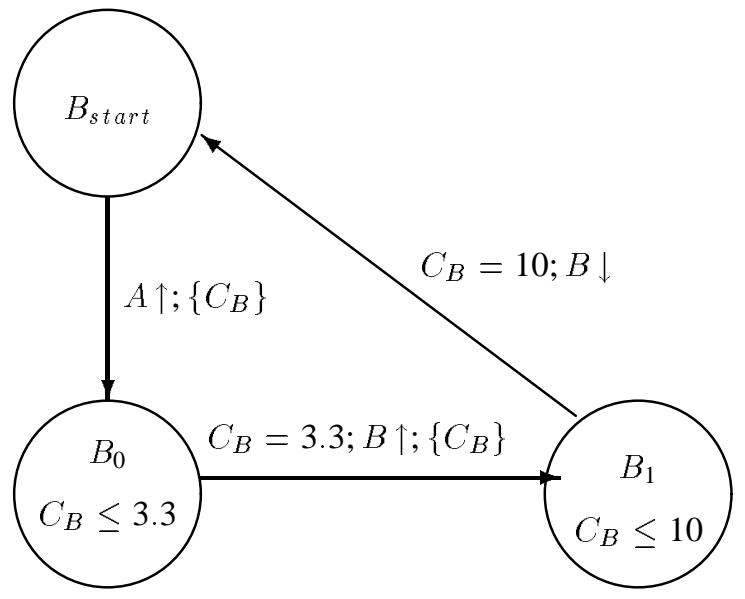

Figure 6. The automata for the input signal B.

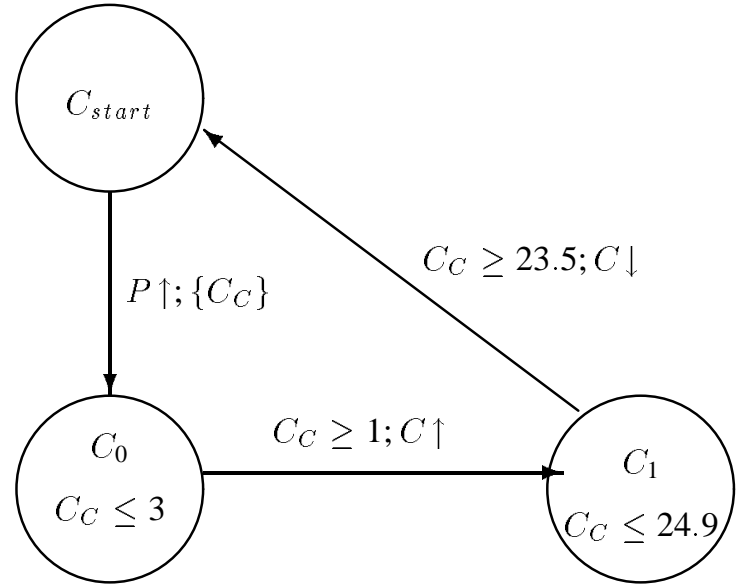

Figure 7. The automata for the input signal $\mathrm{C}$.

As a first step we compose the automata of $P, A, B$, and $C$ with $T_{1}, T_{2}, T_{3}$, and $T_{4}$. This gives us an automaton with 193 states, 529 transitions and 8 clocks. We then define a formula $X$ as

$\left(T_{1}=O n\right) \wedge \neg\left(\left(T_{2}=O n\right) \wedge\left(\left(T_{3}=O n\right) \vee\left(T_{4}=O n\right)\right)\right)$

and apply a special utility that adds the event labels $X \uparrow$ and $X \downarrow$ to all the transitions of the product that change the value of $X$. Then we compose the automaton with the remaining transistors $T_{5}, T_{6}, T_{7}$ and $T_{8}$, and obtain the whole circuit as a timed automaton with 769 states, 2683 transitions and 12 clocks.

The property that we want to verify is the absence of short-cuts, namely current flowing from VDD to GND for all the possible executions starting at the initial state. This is expressed in the formula

$\neg\left(\left(T_{1}=O n\right) \wedge\left(T_{2}=O n\right) \wedge\left(\left(T_{3}=O n\right) \vee\left(T_{4}=O n\right)\right)\right) \wedge$

$\neg\left(\left(T_{5}=O n\right) \wedge\left(T_{6}=O n\right) \wedge\left(\left(T_{3}=O n\right) \vee\left(T_{7}=O n\right)\right)\right)$

We call KRONOS to evaluate this formula against the system and the result is false. In this case we obtain an evaluation file containing a generalized counter-example (appendix 2 ), which indicates classes of timed event sequences that invalidate the formula. From this output one can extract a concrete counter example and deduce which change of parameters will guarantee the satisfaction of the formula. For 
example, in this case a bad sequence of events is:

$$
\begin{aligned}
& (P \uparrow, 0) \rightarrow\left(T_{5} \uparrow, 1.0\right) \rightarrow(C \uparrow, 1.5) \rightarrow\left(T_{4} \uparrow, 2.5\right) \rightarrow \\
& \left(T_{8} \uparrow, 2.5\right) \rightarrow(A \uparrow, 5.0) \rightarrow\left(T_{2} \uparrow, 6.0\right) \rightarrow \\
& (B \uparrow, 8.3) \rightarrow\left(T_{3} \uparrow, 9.3\right) \rightarrow(B \downarrow, 18.3) \rightarrow \\
& \left(T_{3} \downarrow, 18.3\right) \rightarrow(P \downarrow, 20.0) \rightarrow\left(T_{1} \uparrow, 21.0\right)
\end{aligned}
$$

If, on the other hand, we change the system such that $P$ must wait more than 21 time units before falling, we can make sure that $A \downarrow$ and $T_{2} \downarrow$ occur before $T_{1} \uparrow$ and, indeed, when we verify this property with the modified system we get a positive answer from KRONOS. The verification CPU time (on Sun SparcStation 20 with 64MB of memory) was 5 seconds when the property was false and 27 seconds when it was true.

\section{References}

[1] R. Alur, C. Courcoubetis, and D.L. Dill. Model checking in dense real time. Information and Computation, 104(1):2-34, 1993.

[2] R. Alur and D.L. Dill. A theory of timed automata. Theoretical Computer Science, 126:183-235, 1994.

[3] J.R. Burch, E.M. Clarke, K.L. McMillan, D.L. Dill, and L.J. Hwang, Symbolic Model-Checking: $10^{20}$ States and Beyond, Proc. LICS'90, Philadelphia, 1990.

[4] C. Daws, A. Olivero, and S. Yovine. Verifying ET-LOTOS programs with KRONOS. In Proc. FORTE'94, pages 227-242, Bern, Switzerland, October 1994.

[5] C. Daws and S. Yovine. Two examples of verification of multirate timed automata with KRONOS. In Proc. 1995 IEEE RTSS'95, Pisa, Italy, December 1995. IEEE Computer Society Press.

[6] C. Daws, A. Olivero, S. Tripakis, and S. Yovine, The tool KRONOS. In Workshop on Hybrid Systems and Autonomous Control, DIMACS, New Jersey, October 1995.

[7] D. Dill, Timing Assumptions and Verification of Finite-State Concurrent Systems, in J. Sifakis (Ed.), Automatic Verification Methods for Finite State Systems, LNCS 407, Springer, 1989.
[8] E. Clarke and E.A. Emerson, Design and synthesis of synchronization skeletons using branching-time temporal logic, In Workshop on Login of Programs, LNCS $131,1981$.

[9] T.A. Henzinger, X. Nicollin, J. Sifakis, and S. Yovine. Symbolic model checking for real-time systems. Information and Computation, 111(2):193-244, 1994.

[10] K.L. McMillan, Symbolic Model-Checking: an Approach to the State-Explosion problem, Kluwer, 1993.

[11] O. Maler and A. Pnueli, Timing Analysis of Asynchronous Circuits using Timed Automata, in P.E. Camurati, H. Eveking (Eds.), Proc. CHARME'95, 189205, LNCS 987, Springer, 1995. 


\section{Appendix 1}

This is the source code for defining the input signal $A$ and its transistor $T_{2}$. Note that all constant are multiplied by ten.

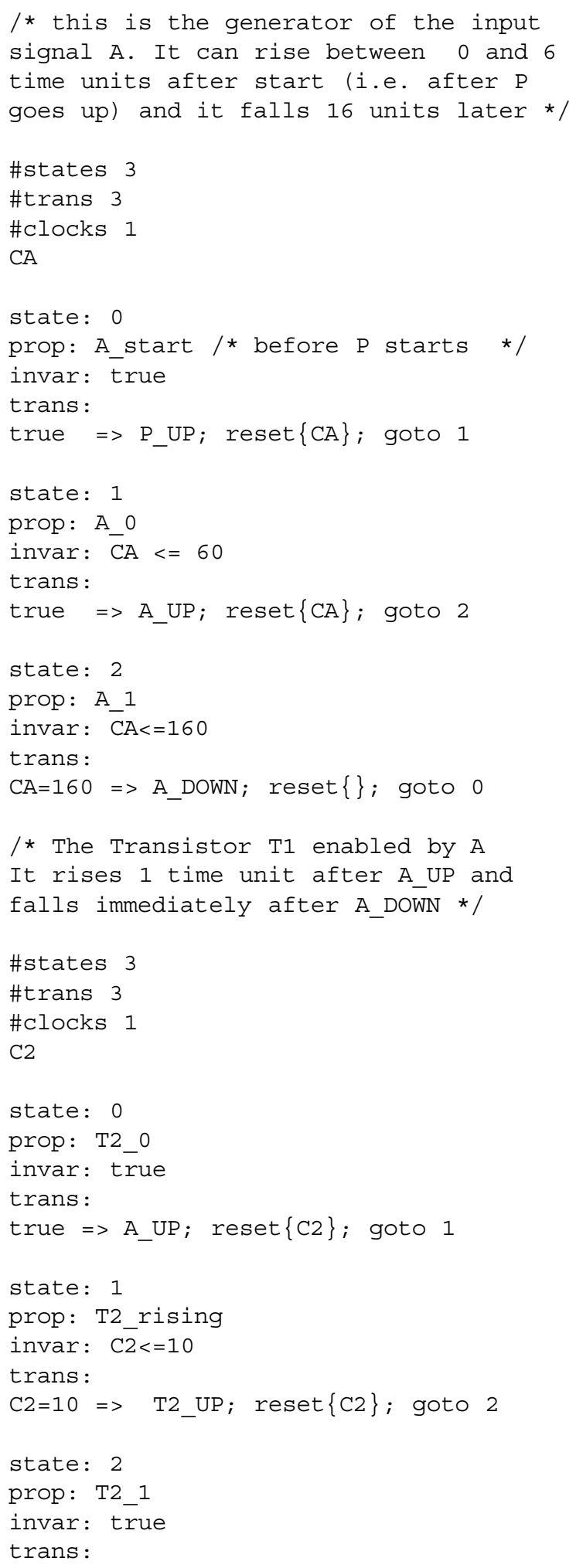

true =>A_DOWN; $\operatorname{reset}\{\} ;$ goto 0

This is the source code for the property we wish to verify:

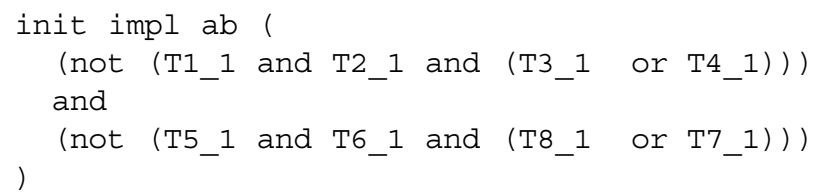

\section{Appendix 2}

This is the beginning and the end of the counter-example information provided by KRONOS when a the verification of a property fails. It is a sequence of generalized states and transitions. A generalized state consists of a location and a set of constraints on the system clocks, including an additional variable $T$ representing the global time. A generalized transition is an event that can take place when certain conditions on the clock are satisfied. From these constraints one can deduce a family of system runs (one of which is described in the body of the paper) which invalidate the property.

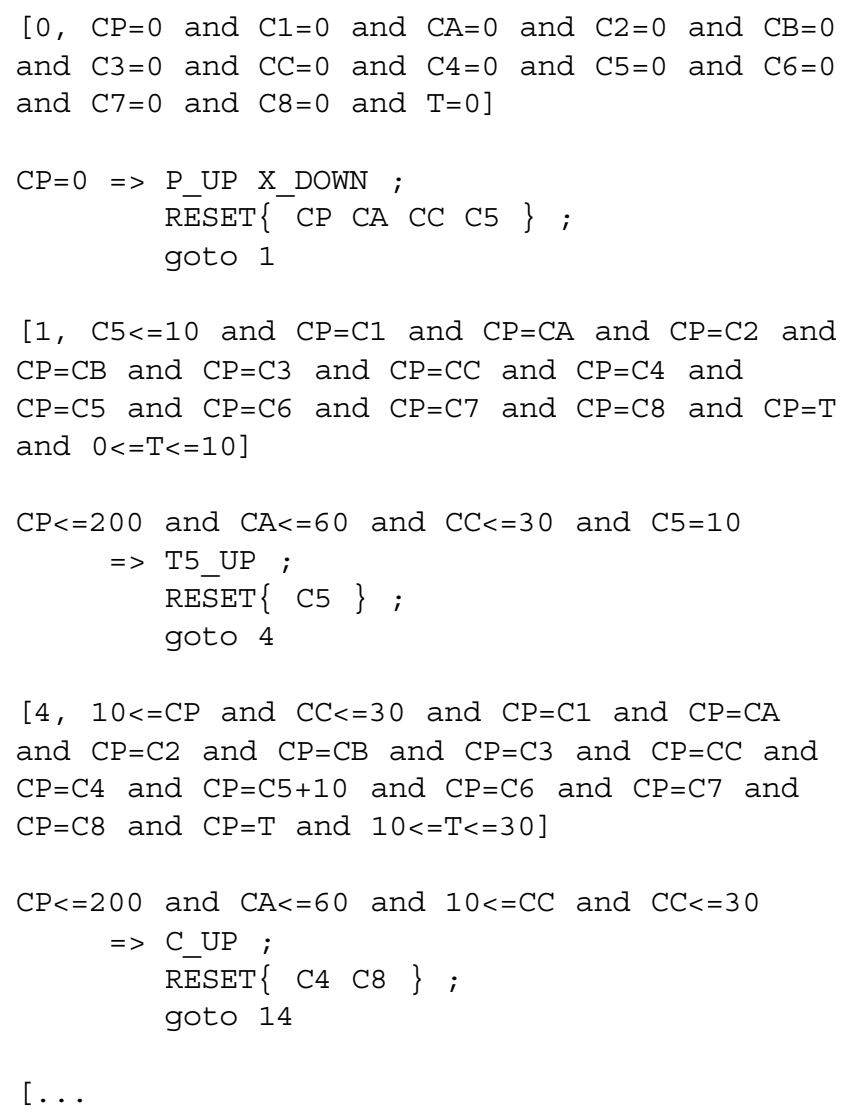


some states and transitions deleted

...]

[442, $\mathrm{CP}<=200$ and $133<=\mathrm{CA}$ and $\mathrm{CA}<=160$ and $\mathrm{CP}=\mathrm{C} 1$ and $\mathrm{CP}<=\mathrm{CA}+60$ and $\mathrm{CP}=\mathrm{CC}$ and $\mathrm{CP}<=\mathrm{C} 4+40$ and $\mathrm{C} 4+20<=\mathrm{CP}$ and $\mathrm{CP}=\mathrm{C} 5+10$ and $\mathrm{CP}=\mathrm{C} 6$ and $\mathrm{CP}=\mathrm{C} 7$ and $\mathrm{CA}=\mathrm{C} 2+10$ and $\mathrm{CA}=\mathrm{CB}+133$ and $\mathrm{CA}=\mathrm{C} 3+43$ and $\mathrm{CA}<=\mathrm{C} 4$ and $\mathrm{C} 4=\mathrm{C} 8$ and and $\mathrm{CP}=\mathrm{T}$ and $153<=\mathrm{T}<=200$ ]

$\mathrm{CP}=200$ and $\mathrm{CA}<=160$ and $\mathrm{CC}<=249$

$=>$ P_DOWN ; $\operatorname{RESET}\{\mathrm{CP} \mathrm{C} 1\}$; goto 474

[474, $\mathrm{C} 1<=10$ and $\mathrm{CA}<=160$ and $\mathrm{CP}=\mathrm{C} 1$ and $\mathrm{CP}+140<=\mathrm{CA}$ and $\mathrm{CP}+200=\mathrm{CC}$ and $\mathrm{CP}+160<=\mathrm{C} 4$ and $\mathrm{C} 4<=\mathrm{CP}+180$ and $\mathrm{CP}+190=\mathrm{C} 5$ and $\mathrm{CP}+200=\mathrm{C} 6$ and $\mathrm{CP}+200=\mathrm{C} 7$ and $\mathrm{CA}=\mathrm{C} 2+10$ and $\mathrm{CA}=\mathrm{CB}+133$ and $\mathrm{CA}=\mathrm{C} 3+43$ and $\mathrm{CA}<=\mathrm{C} 8$ and $\mathrm{C} 4=\mathrm{C} 8$ and $\mathrm{T}=200$ ]

$\mathrm{CP}<=80$ and $\mathrm{C} 1=10$ and $\mathrm{CA}<=160$ and $\mathrm{CC}<=249$ $=>$ T1_UP ; $\operatorname{RESET}\{\}$; goto 516

[516, $10<=\mathrm{CP}$ and $\mathrm{CA}<=160$ and $\mathrm{CP}=\mathrm{C} 1$ and $\mathrm{CP}+140<=\mathrm{CA}$ and $\mathrm{CP}+200=\mathrm{CC}$ and $\mathrm{CP}+160<=\mathrm{C} 4$ and $\mathrm{C} 4<=\mathrm{CP}+180$ and $\mathrm{CP}+190=\mathrm{C} 5$ and $\mathrm{CP}+200=\mathrm{C} 6$ and $\mathrm{CP}+200=\mathrm{C} 7$ and $\mathrm{CA}=\mathrm{C} 2+10$ and $\mathrm{CA}=\mathrm{CB}+133$ and $\mathrm{CA}=\mathrm{C} 3+43$ and $\mathrm{CA}+10<=\mathrm{C} 8$ and $\mathrm{C} 4=\mathrm{C} 8$ and $\mathrm{T}=\mathrm{CP}+200$ and $210<=\mathrm{T}]$ 